\title{
Growth heterogeneity in broiler breeder pullets is settled before the onset of feed restriction but is not predicted by size at hatch 1
}

\author{
C. Lindholm, ${ }^{* 2}$ J. Jönsson, * A. Calais, * A. Middelkoop, $* 3$ \\ N. Yngwe, $\dagger$ E. Berndtson, $\dagger$ J. J. Lees, $*$ E. Hult, $\dagger$ and J. Altimiras* \\ *AVIAN Behavioural Genomics and Physiology group, \\ IFM Biology, Linköping University, 58183 Linköping, Sweden; and †SweHatch AB, 24747 Flyinge, Sweden
}

\begin{abstract}
Uniform growth is a desirable trait in all large-scale animal production systems because it simplifies animal management and increases profitability. In parental broiler flocks, so-called broiler breeders, low growth uniformity is largely attributed to the feed competition that arises from quantitatively restricted feeding. As feed restriction is crucial to maintaining healthy and fertile breeders, several practices for reducing feed competition and the associated growth heterogeneity have been suggested and range from nutrient dilution by increasing fiber content in feed to intermittent fasting with increased portion size ("skip a day"), but no practice appears to be entirely effective. The fact that a large part of the heterogeneity remains even when feed competition is minimized suggests that some growth variation is caused by other factors. We investigated whether this variation arises during embryonic development (as measured by size at hatch) or during posthatch development by following the growth and body composition of birds of varying hatch sizes. Our results support the posthatch alternative, with animals that later grow to be small or large (here defined as $>1$ SD lighter or heavier than mean
\end{abstract}

BW of the flock) being significantly different in size as early as $1 \mathrm{~d}$ after gaining access to feed $(P<0.05)$. We then investigated 2 possible causes for different postnatal growth: that high growth performance is linked 1) to interindividual variations in metabolism (as measured by cloacal temperature and verified by respirometry) or 2) to higher levels of social motivation (as measured in a social reinstatement T-maze), which should reduce the stress of being reared in large-scale commercial flocks. Neither of these follow-up hypotheses could account for the observed heterogeneity in growth. We suggest that the basis of growth heterogeneity in broiler breeder pullets may already be determined at the time of hatch in the form of qualitatively different maternal investments or immediately thereafter as an indirect result of differences in incubation conditions, hatching time, and resulting fasting time. Although this potential difference in maternal investment is not seen in body mass, tarsometatarsal length, or full body length of day-old chicks arriving at the farm, it may influence the development of differential feed and water intake during the first day of feeding, which in turn has direct effects on growth heterogeneity.

Key words: broiler breeders, feed restriction, growth uniformity, ontogenetic growth, poultry, resource allocation

\footnotetext{
${ }^{1}$ This study was funded in its entirety by grant 2013-293 from the Swedish research council Formas to J.A. The authors would like to thank Å. Näsström, M. Elfwing, S. Johansson, M. Öman, F. Östensson, I. Mikkola, and V. Karczmarz for valuable help with experimental work and L. Bektić and D. Ekström for help with animal care.

${ }^{2}$ Corresponding author: caroline.lindholm@liu.se

${ }^{3}$ Present address: Adaptation Physiology Group, Department of Animal Sciences, Wageningen University, P.O. Box 338, 6700 AH Wageningen, The Netherlands.

Received August 19, 2016.

Accepted October 25, 2016.
}

\section{INTRODUCTION}

Growth heterogeneity is a common management and welfare concern in parental broiler flocks. Because these animals need to be feed restricted, reduced growth uniformity has mainly been linked to feed competition (Aviagen, 2013; Zuidhof et al., 2015), but management procedures aimed at reducing competition (e.g., feed scattering, size sorting, intermittent fasting) can, at best, decrease the 


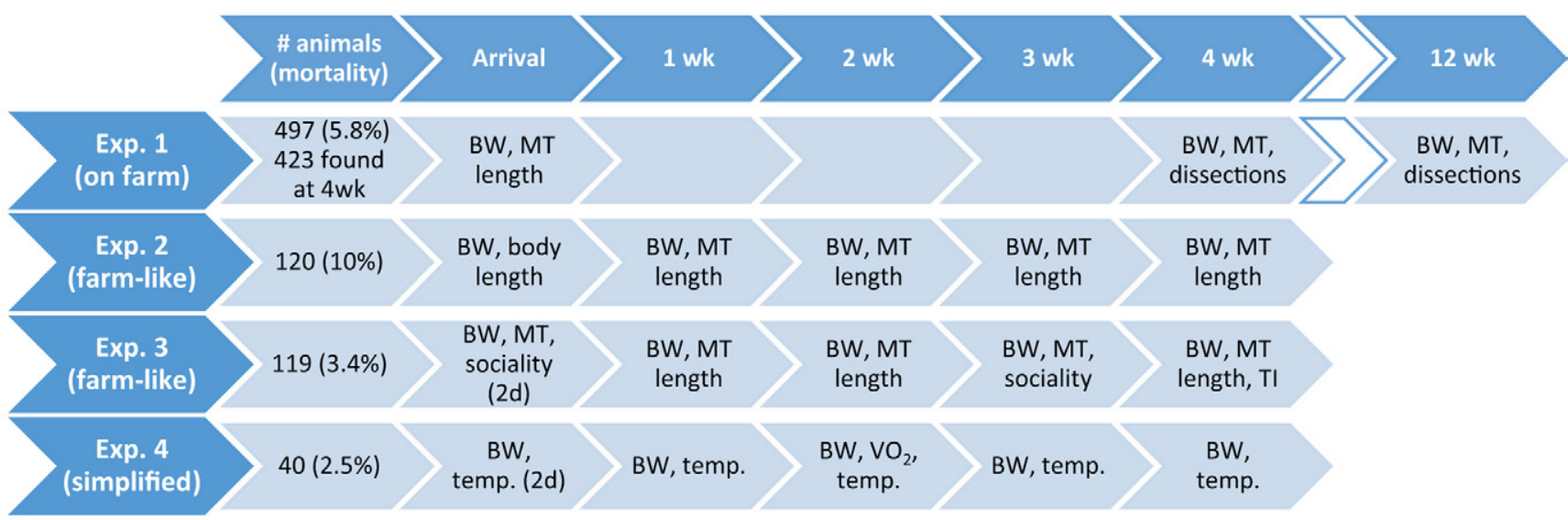

Figure 1. Outline of which data were collected from each of the 4 experiments. All chicks arrived to farm as $1 \mathrm{~d}$ old. In Exp. 2 to 4 the number of animals constitutes all the animals raised in the pen. In Exp. 1, 468 animals survived to 4 wk of age, but 45 of these avoided recapture. It is unknown whether these animals lost their tags, were accidently transferred to other pens, or simply successfully hid within the flock of approx. 3,500 birds. MT $=$ tarsometatarsus, $\mathrm{TI}=$ tonic immobility.

coefficient of variation from $15.3 \%$ to $10.9 \%$ (Zuidhof et al., 2015), indicating that an important part of the variation in growth is independent of feed competition.

In this study we investigated the predictive value of size at hatch and early postnatal growth on the uniformity of broiler breeder pullets under commercial conditions, as it is known that differing embryonic conditions cause variations in metabolism and residual yolk mass (Vieira and Moran, 1998; Joseph et al., 2006). Although size at hatch is a poor indicator of future growth in broilers fed ad libitum (Willemsen et al., 2008), it is not known to what extent feed restriction reduces opportunities for compensatory growth, potentially increasing the importance of hatch size.

We also explored 2 mechanisms that could potentially explain differences in posthatch growth rates. First, highly social individuals may experience reduced stress when raised in large flocks. We have previously reported a possible correlation between sociality and growth performance in broiler breeders (Lindholm et al., 2015), and others have shown it in ad libitum-fed broilers (Marin et al., 1999, 2003). Second, variations in the development of thermoregulatory capacity have previously been linked to early growth performance (Peebles et al., 2004) and may have long-term effects on metabolic rate and growth.

\section{MATERIALS AND METHODS}

The data presented in this paper were obtained from 4 different experiments tracking the growth of young broiler-type chickens. A brief overview of the different experiments and the data collection performed is presented in Fig. 1. All animal experiments were approved by the regional ethical committee of Malmö/Lund (diary number M 71-14).

\section{Animal Rearing}

The animals used in these experiments were female Ross 308 broiler breeders raised on a commercial breeder rearing farm (Exp. 1) or under farm-like conditions, mimicking commercial stocking density and management procedures (i.e., lighting, temperature, handling, feeding system, and quality) in a lab facility (Exp. 2-3). Experiment 4 was performed under simplified conditions, raising Ross 308 broilers of mixed sexes in a lab setting. All animals were raised on wood shavings and feed restricted according to supplier guidelines (Aviagen, 2013). The number of animals included and total mortality for each experiment can be found in Fig. 1. In each experiment, all birds were hatched from the same (grand-)maternal flock. Grandmaternal ages were $30 \mathrm{wk}$ (Exp. 1), $33 \mathrm{wk}$ (Exp. 3), and 45 wk (Exp. 2). Maternal age in Exp. 4 was 34 wk.

Farm-raised animals were part of a flock of approximately 3,500 hens at a density of approx. 15 birds $/ \mathrm{m}^{2}$ until grading (size sorting) at $4 \mathrm{wk}$ of age. Commercial stocking density was matched in the farm-like experiment. On farm and in the farm-like experiments identical commercial-grade feed was provided from several round troughs or on paper on $\mathrm{d} 1$ and gradually substituted (at 8 to $13 \mathrm{~d}$ ) with an automated feeder system that distributed the feed throughout the pen 15 min after dawn each morning. Two starter feeds were given, the first one until d $9(12 \mathrm{MJ} / \mathrm{kg}, 22 \% \mathrm{CP})$ and the subsequent one for the remainder of the experiment $(11.4 \mathrm{MJ} /$ $\mathrm{kg}, 18 \%$ to $19 \% \mathrm{CP}$ ). Water was available ad libitum in all experiments. Lighting was gradually reduced from $23 \mathrm{~h}$ and 22 lux on $\mathrm{d} 1$ to $8 \mathrm{~h}$ and 5 lux on d 14, with each new light period starting at $0700 \mathrm{~h}$. Temperature was gradually decreased from $33^{\circ} \mathrm{C}$ on $\mathrm{d} 1$ to $20^{\circ} \mathrm{C}$ on d 22. For Exp. 2 and 3, growth in the farm simulations was also compared to weight data reported from the 
respective farms rearing breeder pullets from the same batch to verify that similar growth rates were achieved.

In the more simplified Exp. 4, animals were reared in similar conditions at a stocking density of $10 \mathrm{birds} / \mathrm{m}^{2}$. Feed restriction was in place, with feed manually scattered across the pen at $0800 \mathrm{~h}$ each morning, and water was available ad libitum from bell drinkers. The same type of feed (11 MJ/kg, 19\% CP) was given throughout the experiment. Lighting was on a fixed 12L:12D cycle (light 0700 to 1900) with an intensity of approx. 15 lux, but temperature was kept similar to that in the previous experiments. Both males and females were included in the analyses, with no significant effect of sex.

\section{Growth Monitoring}

All animals were weighed and wing banded on arrival at the rearing facility. This weight will be referred to as hatch weight as animals had not previously had access to feed or water. Animals were then weighed on a weekly basis at the experimental facilities (Exp. 2 to 4 ) or at 4 and $12 \mathrm{wk}$ of age at the commercial farm (Exp. 1). Weighing was performed after the animals were fed, with the exception of arrival at the rearing facility and BW measurements collected in conjunction with morning temperature recordings in Exp. 4. Weekly weights were used to calculate the average weekly growth rate (reported as g/d) in Exp. 2 to 4 . In each experiment, animals were classified as small (SA) or large (LA) for being >1 SD smaller or larger, respectively, than the mean BW of the flock at $4 \mathrm{wk}$ of age. In addition to BW, tarsometatarsal (MT) length (outside of hock to underside of foot pad) was measured by digital calipers during Exp. 1 to 3, and full body length (from tip of beak to end of middle toe when fully stretched) was measured at the start of Exp. 2 . All length-based measurements were performed by the same experimenter to ensure consistent recordings.

\section{Behavioral Tests}

The social motivation of all chicks in Exp. 3 was tested using a social reinstatement T-maze as previously described by Marin et al. (1999). Chicks were first tested at $2 \mathrm{~d}$ of age and then retested at $3 \mathrm{wk}$ of age. During each test, the chicks were randomly assigned to 1 of 4 to 6 test groups (maximum of 20 animals per group at $2 \mathrm{~d}$, maximum of 30 animals per group at $3 \mathrm{wk}$ ). Before the test, each test group was acclimatized for $15 \mathrm{~min}$ to a brooding area $(60 \times 60 \mathrm{~cm}$ at $2 \mathrm{~d}, 90 \times 90 \mathrm{~cm}$ at $3 \mathrm{wk})$ outfitted with wood shavings and a water bell. At $2 \mathrm{~d}$ of age, the brooding area also contained a feed trough and was heated by a heat lamp. At the start of the test, 1 chicken at a time was removed from its test group and placed in the T-maze start box $(21 \times 21 \mathrm{~cm}$ or $30 \times 30 \mathrm{~cm}$ depending

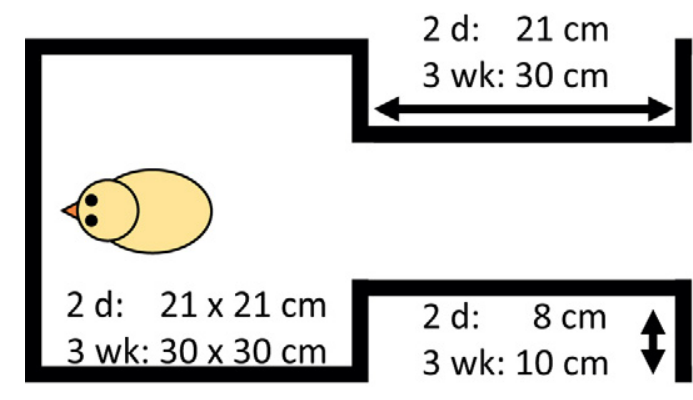

Figure 2. Schematic drawing of the sociality T-maze used and its measurements when animals were tested at $2 \mathrm{~d}$ and $3 \mathrm{wk}$ of age, respectively. Placement and orientation of the chick at start of the test are indicated. The brooding area was reachable through either a left or right (randomized between tests) turn at the mirror section.

on age), facing the back wall (Fig. 2). Emergence from the start box was encouraged by placing a mirror $(10 \times$ $10 \mathrm{~cm}$ ) at the T-maze junction $(21$ or $30 \mathrm{~cm}$ from start box, respectively). Chicks were scored for their latency to exit the start box, latency to reach the mirror section, and latency to exit the T-maze and return to the brooding area through the correct arm $(8$ or $10 \mathrm{~cm}$ long, respectively). An animal that performed 3 consecutive escape attempts in the maze was returned to its test group and retested later. Each animal was given up to 3 such trials (a maximum of 9 escape attempts in total), with all animals successful at $2 \mathrm{~d}$ and 9 animals excluded from analysis at $3 \mathrm{wk}$. Animals were categorized as high performers (HP) or low performers (LP) for being among the $25 \%$ quickest or slowest, respectively, to reach the mirror section, according to the recommendation of Marin et al. (2003).

At the end of Exp. 3, all animals that had been categorized as either HP or LP on the basis of maze performance, or, alternatively, SA or LA on the basis of growth performance, were subjected to a tonic immobility (TI) test. Tonic immobility was induced by $15 \mathrm{~s}$ of manual back restraint, and each bird was given up to 3 induction trials. Tonic immobility was interrupted after $5 \mathrm{~min}$. Two measurements of TI duration were recorded: latency to start moving the head and latency to righting ("total duration").

\section{Body Temperature and Basal Metabolic Rate}

Cloacal temperature was measured in all animals on several days each week during Exp. 4. Animals were collected from the pen at $0700 \mathrm{~h}$ (at dawn, before feeding), $1400 \mathrm{~h}$, and $2100 \mathrm{~h}$ ( $2 \mathrm{~h}$ after dark) and kept at an ambient temperature of approximately $26^{\circ} \mathrm{C}$ during the measurements. During the first week, the chicks were kept under a heat lamp to raise ambient temperature to approx. $33^{\circ} \mathrm{C}$. Cloacal temperature was measured by inserting a standard medical thermometer approx. $7 \mathrm{~mm}$ into the cloaca of each animal. All measurements were performed by the same experimenters to ensure consistent recordings. 
At $16 \mathrm{~d}$ of age, the 6 smallest (200.6 to $206.2 \mathrm{~g}$; SA $<213.3 \mathrm{~g}$ ) and 6 largest (261.3 to $281.5 \mathrm{~g}$; LA $>261.9 \mathrm{~g}$ ) chickens in Exp. 4 were chosen for metabolic measurements. Oxygen consumption was measured overnight in respiration chambers when the animals were 16 to $19 \mathrm{~d}$ old as previously described (Lindgren and Altimiras, 2013). Briefly, 4 animals were individually placed in 3-L respirometers, and measurements were taken overnight ( $1700 \mathrm{~h}$ to $0700 \mathrm{~h}$ ) in a temperature-controlled climatic chamber (Rumed Cooled Incubator Model 3001, Rubarth Apparate $\mathrm{GmBH}$, Laatzen, Germany) set at $32^{\circ} \mathrm{C}$. Experimental support for this temperature representing the thermoneutral zone is presented in Appendix 1. Air flow through the respirometers was set at $1 \mathrm{~L} / \mathrm{min}$ (Flow Bar, Sable Systems, Las Vegas, NV). Gas from the 4 respirometers was analyzed using an oxygen analyzer (FOXBOX II, Sable Systems) for $2.5 \mathrm{~min}$ per channel, resulting in 1 measurement for each animal every $10 \mathrm{~min}$. Before entering the gas analyzer, the air passed through Drierite (calcium sulfate with cobalt chloride; Hammond Drierite, Xenia, $\mathrm{OH}$ ) to remove water vapor. Baseline $\mathrm{O}_{2}$ levels (related to room $\mathrm{O}_{2}$ and sensor drift) were recorded between every chamber measurement and accounted for at the time of calculation. Oxygen consumption was calculated from the standard equation in the absence of $\mathrm{CO}_{2}$ scrubbing (Eq. [10.2] in Lighton, 2008). Basal metabolic rate was obtained from the smallest oxygen consumption value over $60 \mathrm{~min}$ from running averages of 6 consecutive point measurements. All values are presented as mass-specific oxygen consumption $\left(\mathrm{mL} \mathrm{O}_{2} \cdot \mathrm{g}^{-1} \cdot \mathrm{h}^{-1}\right)$.

\section{Body Composition}

Dissections were performed on forty-five 4-wkold animals and twenty-eight 12-wk-old animals from Exp. 1. Four-week-old animals were chosen for being either small or large at hatch and at $4 \mathrm{wk}$ of age in a $2 \times 2$ experimental design, aiming for 12 animals per group (cf. Lindholm et al., 2015). Three animals turned out to be male on dissection and were discarded, resulting in 12 animals that were hatched small and grew small (SS), 10 animals that hatched small and grew large (SL), 12 animals that hatched large and grew large (LL), and 11 animals that hatched large and grew small (LS) to be used for analyzing the effects of hatch size on later body composition. Hatch and 4-wk weights of the 4 groups are shown in Fig. 3D.

Hens chosen for dissections were rendered unconscious by a blow to the head and euthanized by decapitation; the gastrointestinal tract, heart, and liver were removed, and all organs and the carcass were then packed separately in dry ice for transport and moved to $-30^{\circ} \mathrm{C}$ within $12 \mathrm{~h}$. Organs and carcasses were thawed in $+4^{\circ} \mathrm{C}$ overnight before being dissected further. Organs (ventricular heart, liver, lung, kidney, crop, proventriculus, gizzard, pancreas, spleen, and bursa fabricii) and muscles (pectoralis, iliotibialis, gastrocnemius) were weighed for blotted wet mass, and the fat surrounding the proventriculus and gizzard (reported as abdominal fat) was collected and weighed as an indicator of adiposity. The mass of the ventricles was recorded as heart mass. For muscles and bilateral organs (lungs and kidneys), only the left muscle or organ was dissected out and weighed. Lean BW was estimated by subtracting the weight of residual feed in the crop, proventriculus, and gizzard as well as abdominal fat from the weight of the intact animal.

At 12 wk of age, 14 hens that had been graded as small $(1,167$ to $1,454 \mathrm{~g})$ and 14 hens graded as normal/ large $(1,473$ to $1,650 \mathrm{~g})$ were chosen for dissections. Dissections were performed in the same way as for the 4-wk birds. Two large animals turned out to be male on dissection and were discarded from further analysis.

\section{Statistical Analysis}

Throughout this study, the individual bird was used as a statistical unit as the goal was to account for individual differences in growth. Permutation tests were used to compare the means of 2 groups as these make fewer assumptions than $t$ tests and are valid even if the population distribution is nonnormal and sampling is not strictly random (Lew, 2008). Although modern computing has made 2-group permutation testing accessible, comparisons of more than 2 groups using this method are computationally unfeasible (Cousineau, 2011); thus, ANOVA were performed for testing the difference of more than 2 groups. Permutation tests were performed using a Boss Permutations Tester (Michael J. Lew, Department of Pharmacology, University of Melbourne). Note that permutation tests produce no test statistic other than the $P$-value. Analyses of variance, survival analysis (for comparing TI latencies), and correlation analyses were performed in Minitab 17 (Minitab Inc., State College, PA). Log-rank tests were used to determine statistically different distributions of behavioral latencies and the number of induction trials between groups. Results were considered statistically significant when $P<0.05$. Where present, error bars denote SD.

\section{RESULTS}

Body weight at hatch $(1 \mathrm{~d})$ showed significant correlation with 4 -wk BW in 2 out of 4 trials $(P \leq 0.001$; Fig. 3A) but could explain only a small part of the variation in size at the older age $(7.7 \%$ and $9.5 \%$ in Exp. 1 and 2, respectively; Table 1). Body weight at $1 \mathrm{wk}$ of age was consistently a better predictor of continued 

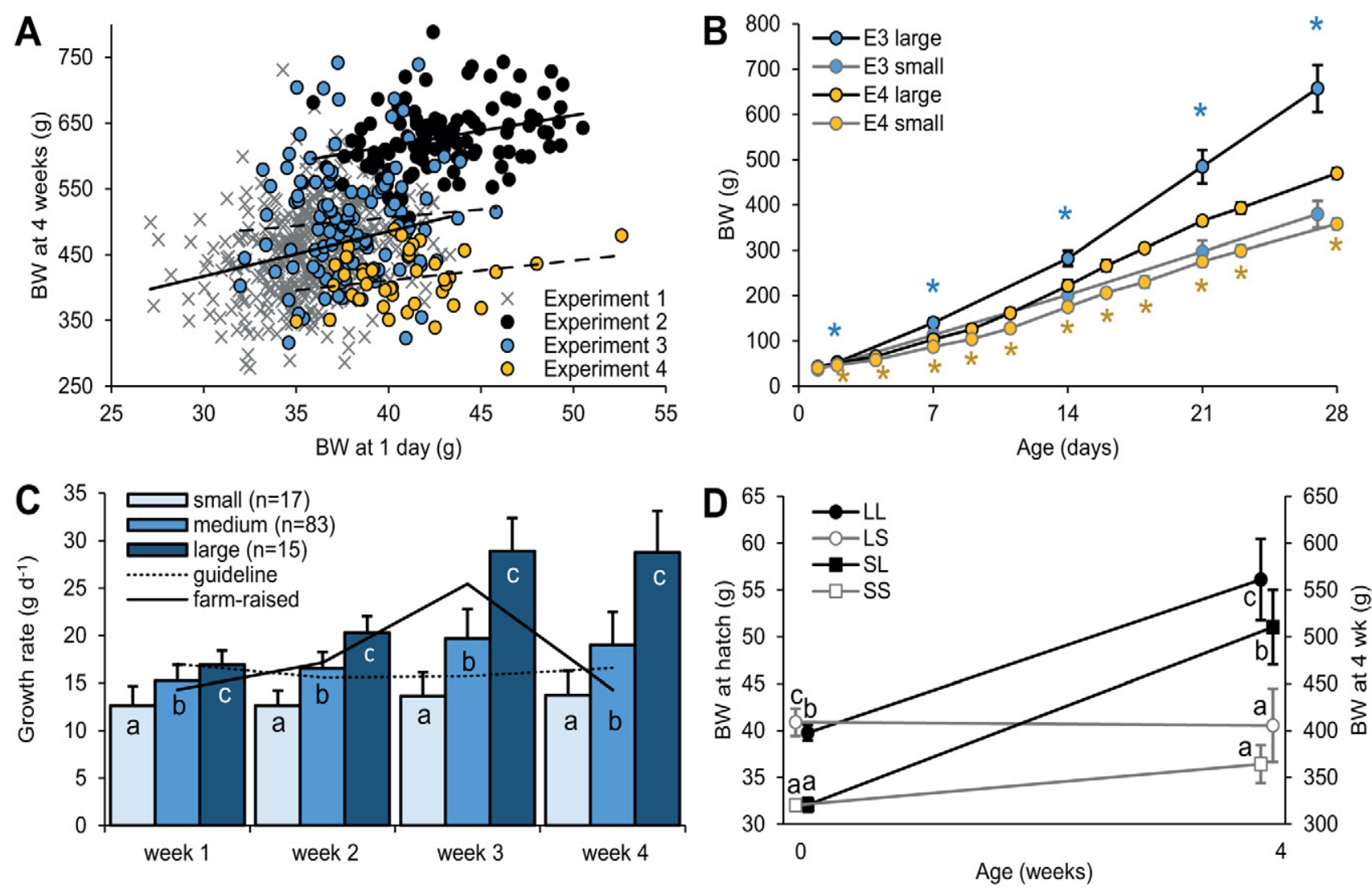

Figure 3. (A) Hatch weight is significantly correlated with 4-wk weight in Exp. 1 and 2 (solid trend lines), although correlations are weak (see Table 1). The 2 weights are not significantly correlated in Exp. 3 and 4 (dashed trend lines). (B) Growth trajectories of large (black lines) and small (gray lines) 4-wk animals in Exp. 3 (blue, 17 large and 15 small animals) and Exp. 4 (yellow, 8 animals per group). In both cases, large and small animals are significantly different from $2 \mathrm{~d}$ of age but not at hatch. (C) Weekly growth rates during Exp. 3 split into small, intermediate, and large birds. Small birds ( $>1$ SD smaller than mean at 4 wk) grow at a consistently low growth rate, whereas intermediate and large birds start at higher growth rates that then increase significantly during the 4-wk period. Different letters denote significantly different growth rates within each week. The solid line represents growth reported for chicks from the same hatch group raised on a commercial breeder rearing farm. The dashed line is derived from official Aviagen guidelines for this breed. (D) Weight development over the 4-wk growth period for dissected birds from Exp. 1. Birds were selected on hatch weight and 4-wk weight as being either small or large. Different letters denote significant differences within that age as determined by ANOVA with post hoc Tukey comparison $(F=258.19, P<0.001$ at hatch; $F=66.09, P<0.001$ at 4 wk). All error bars denote SD.

growth performance, explaining $35.9 \%$ to $55.4 \%$ of the variation, but was not available for the farm-raised chickens. The 2 length-based measurements used at hatch, full body length and MT length, were consistently worse predictors of growth than hatch weight (Table 1). Hatch weights varied between experiments, with differences in maternal age accounting for $45.5 \%$ of the overall variation between breeder hatchlings $(P<0.001$, data not shown), and experiments were thus not pooled for analysis.

In all farm simulations, chicks that grew to a large or small size at 4 wk were already significantly different at the first weighing after being introduced to feed, as early as $2 \mathrm{~d}$ of age $(P<0.0001$ and $P=0.019$ in Exp. 3 and 4, respectively; Fig. 3B). Furthermore, small animals showed a consistently lower growth rate throughout the early rearing period compared to both intermediate- and large-sized birds. Large animals grew at higher rates from the first week $(16.9 \mathrm{vs} .12 .6 \mathrm{~g} / \mathrm{d}$ for the small animals; $P<0.0001)$ and also increased their growth rate significantly ( $P=0.0001$ comparing high- est vs. lowest weekly growth rate in large birds) during the first $4 \mathrm{wk}$ of life, something that did not happen for the smaller animals $(P=0.25)$, with the large animals reaching a peak growth rate more than twice that of the small animals ( 28.9 vs. $13.7 \mathrm{~g} / \mathrm{d}$ during the third week of life; $P<0.0001$ ), as shown in Fig. 3C. Results shown are from Exp. 3, which best matched the growth rate recorded on farm for a parallel flock. Experiment 2 produced equivalent results (data not shown).

No difference in body temperature was found between small and large animals, with the exception of the morning measurement at $\mathrm{d} 21$ when small animals were, on average, $0.21^{\circ} \mathrm{C}$ cooler than their large conspecifics, a difference that we deem irrelevant from a physiological standpoint (data not shown). At all time points, temperatures of both groups fell within $1 \mathrm{SD}$ from the mean of intermediate-sized birds (Fig. 4 shows temperatures at $1400 \mathrm{~h}$ ). In line with this, there was no difference in mass-specific basal metabolic rate as determined by oxygen consumption at $2.5 \mathrm{wk}$ of age $(P$ $=0.98)$. Mass-specific baseline oxygen consumption 
Table 1. Correlations between various growth measurements during the first 4 wk of life ${ }^{1}$

\begin{tabular}{|c|c|c|c|c|c|}
\hline \multirow[b]{2}{*}{ Item } & \multirow[b]{2}{*}{ Body length, $1 \mathrm{~d}$} & \multirow[b]{2}{*}{ MT length, $1 \mathrm{~d}$} & \multicolumn{3}{|c|}{ BW } \\
\hline & & & $1 \mathrm{~d}$ & $2 \mathrm{~d}$ & $1 \mathrm{wk}$ \\
\hline $\mathrm{BW}, 1 \mathrm{~d}$ & $\begin{array}{l}\text { E1: n/a } \\
\text { E2: } 0.055,0.016 \\
\text { E3: n/a } \\
\text { E4: n/a }\end{array}$ & $\begin{array}{l}\text { E1: } 0.120,<0.001 \\
\text { E2: } \mathrm{n} / \mathrm{a} \\
\text { E3: } 0.249,<0.001 \\
\text { E4: } \mathrm{n} / \mathrm{a}\end{array}$ & & & \\
\hline $\mathrm{BW}, 2 \mathrm{~d}$ & $\begin{array}{l}\text { Not available for } \\
\text { any experiment }\end{array}$ & $\begin{array}{l}\text { E1: } \mathrm{n} / \mathrm{a} \\
\text { E2: } \mathrm{n} / \mathrm{a} \\
\text { E3: } 0.284,<0.001 \\
\text { E4: } \mathrm{n} / \mathrm{a}\end{array}$ & $\begin{array}{l}\text { E1: } \mathrm{n} / \mathrm{a} \\
\text { E2: } \mathrm{n} / \mathrm{a} \\
\text { E3: } 0.426,<0.001 \\
\text { E4: } 0.677,<0.001\end{array}$ & & \\
\hline BW, 1 wk & $\begin{array}{l}\text { E1: n/a } \\
\text { E2: } 0.081,0.003 \\
\text { E3: n/a } \\
\text { E4: n/a }\end{array}$ & $\begin{array}{l}\text { E1: } \mathrm{n} / \mathrm{a} \\
\text { E2: } \mathrm{n} / \mathrm{a} \\
\text { E3: } 0.057,0.010 \\
\text { E4: } \mathrm{n} / \mathrm{a}\end{array}$ & $\begin{array}{l}\text { E1: } \mathrm{n} / \mathrm{a} \\
\text { E2: } 0.358,<0.001 \\
\text { E3: } 0.111,<0.001 \\
\text { E4: } 0.461,<0.001\end{array}$ & $\begin{array}{l}\text { E1: } \mathrm{n} / \mathrm{a} \\
\text { E2: } \mathrm{n} / \mathrm{a} \\
\text { E3: } 0.207,<0.001 \\
\text { E4: } 0.581,<0.001\end{array}$ & \\
\hline BW, 4 wk & $\begin{array}{l}\text { E1: n/a } \\
\text { E2: ns } \\
\text { E3: n/a } \\
\text { E4: n/a }\end{array}$ & $\begin{array}{l}\text { E1: } 0.021,0.002 \\
\text { E2: n/a } \\
\text { E3: ns } \\
\text { E4: n/a }\end{array}$ & $\begin{array}{l}\text { E1: } 0.077,<0.001 \\
\text { E2: } 0.095,0.001 \\
\text { E3: ns } \\
\text { E4: ns }\end{array}$ & $\begin{array}{l}\text { E1: } \mathrm{n} / \mathrm{a} \\
\text { E2: } \mathrm{n} / \mathrm{a} \\
\text { E3: } 0.039,0.035 \\
\text { E4: } 0.169,0.009\end{array}$ & $\begin{array}{l}\text { E1: } \mathrm{n} / \mathrm{a} \\
\text { E2: } 0.432,<0.001 \\
\text { E3: } 0.359,<0.001 \\
\text { E4: } 0.554,<0.001\end{array}$ \\
\hline Growth, g/d & $\begin{array}{l}\text { E1: n/a } \\
\text { E2: ns } \\
\text { E3: n/a } \\
\text { E4: n/a }\end{array}$ & $\begin{array}{l}\text { E1: } 0.018,0.006 \\
\text { E2: n/a } \\
\text { E3: ns } \\
\text { E4: n/a }\end{array}$ & $\begin{array}{l}\text { E1: } 0.057,<0.001 \\
\text { E2: } 0.062,0.011 \\
\text { E3: ns } \\
\text { E4: ns }\end{array}$ & $\begin{array}{l}\text { E1: } n / a \\
\text { E2: } n / a \\
\text { E3: } 0.031,0.058 \\
\text { E4: } 0.122,0.029\end{array}$ & $\begin{array}{l}\text { E1: } \mathrm{n} / \mathrm{a} \\
\text { E2: } 0.396,<0.001 \\
\text { E3: } 0.348,<0.001 \\
\text { E4: } 0.491,<0.001\end{array}$ \\
\hline
\end{tabular}

\footnotetext{
${ }^{1}$ The results are presented in the form "Experiment number: $r^{2}, P$-value" and include all correlations with $P<0.1$. Length-based measurements in day-old chicks appear to be consistently worse predictors of growth than day-old BW. Here MT $=$ tarsometatarsus; $\mathrm{n} / \mathrm{a}=$ not available; ns $=$ not significant $(P>0.1)$.
}

averaged $1.00 \mathrm{~mL} \mathrm{O} \cdot \mathrm{g}^{-1} \cdot \mathrm{h}^{-1}$ for the small animals and $1.02 \mathrm{~mL} \mathrm{O} \cdot \mathrm{g}^{-1} \cdot \mathrm{h}^{-1}$ for the large animals $(P=0.98)$. Absolute $\mathrm{VO}_{2}$ was $40 \%$ higher in the large animals, but this difference was almost entirely explained by the difference in body mass $\left(r^{2}=0.859 ; P<0.0005\right)$.

T-maze performance of broiler breeder chicks at $2 \mathrm{~d}$ showed no correlation with BW at any age or with overall growth rate (data not shown) and did not predict their performance in the same test at $3 \mathrm{wk}$ of age $\left(r^{2}=\right.$ $0.004, P=0.51$; Fig. 5A). Performance in the maze at 3 wk showed a weak correlation with BW at $4 \mathrm{wk}$, with the HP hens weighing, on average, $60 \mathrm{~g}$, or approximately $11 \%$, less than their least social (LP) conspecifics (Fig. 5B); however, most of these birds were within $1 \mathrm{SD}$ from the mean, and thus, there was not much overlap with our weight-based groups of interest. The overall correlation between 3-wk T-maze performance and growth was weak, although significant $\left(r^{2}=0.076\right.$, $P=0.004$; Fig. 5C). The TI durations were not significantly correlated with sociality (data not shown), and although there was no difference in total duration to righting, small birds were significantly quicker to start moving their heads $(P=0.001$; Fig. 5D). The TI induction was also significantly more successful in the large animals, with $87 \%$ entering TI within 3 induction trials, compared to $53 \%$ of the small animals $(P=0.034)$.

Small and large animals differed in body composition at $4 \mathrm{wk}$ of age, with small hens exhibiting relatively larger hearts $(P=0.036)$, lungs $(P=0.026)$, crops $(P=$ $0.033)$, gizzards $(P=0.036)$, and pancreases $(P=0.011$; Fig. 5A). Larger hens instead had larger muscles and a numerically larger amount of abdominal fat that approached significance $(P=0.071 ;$ Fig. $6 b)$. There was no significant effect of hatchling mass on 4-wk body composition (Table 2). Follow-up dissections of 12-wk animals from the same batch of hatchlings showed that large and small animals were no longer different in terms of leg muscles (iliotibialis and gastrocnemius) and pancreas and gizzard mass and the difference in relative mass of the pectoralis muscle was reduced, although still significant $(P=0.045$; Fig. $6 \mathrm{C}$ and $6 \mathrm{D})$. The smaller animals retained their larger supply organs with larger hearts $(P=$ $0.0016)$, lungs $(P=0.030)$, proventriculi $(P=0.044)$, and crops $(P=0.010$; Fig. $6 \mathrm{C})$. Although the animals had become more similar in overall size, the difference in heart and lung size between the 2 groups increased with age.

\section{DISCUSSION}

The restricted feeding regimes that broiler breeders are subjected to are well known to induce growth heterogeneity, which is typically attributed to increased feed competition (Zuidhof et al., 1995; Aviagen, 2013). Nevertheless, the best predictor of continued growth performance and slaughter weight in ad libitum-fed broilers is the BW at 1 wk of age, which is sometimes considered the true starting point of production (Tona et al., 2003; Decuypere and Bruggeman, 2007). The first week posthatch is crucial as the chick is using up its residual yolk (until around $4 \mathrm{~d}$ of age) and investing most of the yolk into intestinal development (Noy and Sklan, 1999; Lilburn and Loeffler, 2015). Toward the end of this week, the small intestine and 


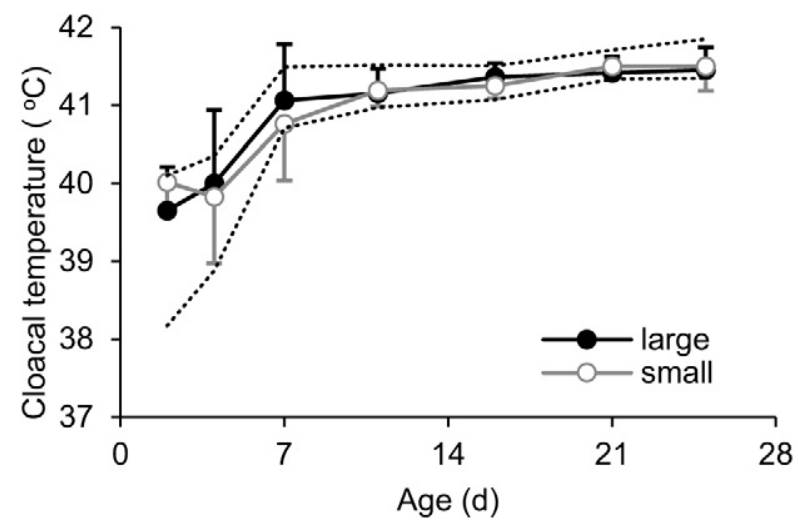

Figure 4. Cloacal temperature of large vs. small animals ( 8 per group) at midday $(1400 \mathrm{~h})$. Dotted lines outline $1 \mathrm{SD}$ from the mean of intermediately sized birds. Recorded temperatures do not differ significantly between groups at any time point shown here. Error bars denote SD.

pancreas will be at their peak growth rates, a microflora will have been established, and the animal will have started relying on its own immune system as maternal IgAs have declined (Lilburn and Loeffler, 2015). Considering that feed restriction in broiler breeders is gradually introduced around $1 \mathrm{wk}$ of age, the importance of early development cannot be underestimated and is likely to account for a significant part of the growth heterogeneity seen later in life. This is further supported by the restricted impact of competition-reducing strategies in improving growth uniformity (Zuidhof et al., 2015).

We provide evidence that BW within a day of first feeding shows a significant correlation with 4-wk BW even when BW at hatch does not. This difference is conserved by differential growth rates, with small animals growing at consistently lower rates. Although the overall growth rate is certainly affected by feed restriction, the differences in growth rate are retained for the full 4-wk period studied here. It has been proposed previously that appetite (or feed consumption), water consumption, and yolk use are the determining factors for growth potential in young chicks (Pinchasov, 1991; Marin et al., 1999), and only differences in feed and water intake are likely explanations for the higher weight gain (up to $40 \%$ in Exp. 4) already seen during the first day of feeding in animals later growing to be large at $4 \mathrm{wk}$. It is worth noting that in both domestic and wild birds with less extreme feeding patterns, egg and/or hatch weight is the determining factor for BW up to $4 \mathrm{~d}$ (Smith and Wettermark, 1995; Løtvedt and Jensen, 2014). Some authors have suggested that length-based measurements such as full body length or shank length are better performance predictors than hatching BW (Wolanski et al., 2006), but we found all hatchling-based measurements to be poor predictors of later growth (cf. Willemsen et al., 2008).

Although early growth is a well-known predictor of future performance, fairly little is known about the mechanisms that promote or demote early growth in certain individuals. It was hypothesized previously that highly social and less fearful animals experience a better welfare situation in large-scale flocks, with improved growth as less energy is directed toward stress and fear responses (Kikusui et al., 2006; Siegel et al., 2008). Comparing the performance of individual chicks in a sociality T-maze at just 2 to $3 \mathrm{~d}$, Marin et al. (1999, 2003) found a positive correlation between early social behavior and later slaughter weight that was reproduced over a range of environments. In our study, T-maze performance at $2 \mathrm{~d}$ showed no correlation with growth at any age or with performance in the same maze at 3 wk of age. Maze performance at 3 wk showed a weak negative correlation with growth to $4 \mathrm{wk}$, but this may have been confounded by the fact that HP and LP pullets were already diverging in growth at the time of the test. Although performance in the maze is expected to primarily reflect sociality, the results may also indicate different levels of fearfulness. We have previously reported shorter TI durations and lower inducibility in small broiler breeders compared to large pen mates at 4 wk (Lindholm et al., 2015), and a similar pattern was found in this study. Performance in the maze, however, was not correlated with TI, and we found no noticeable effect on growth of either fearfulness or sociality. It is possible that the small effect (HP 2.3\% heavier than LP at $8 \mathrm{wk} ; P<0.02)$ of sociality on growth reported by Marin et al. (2003) is masked in feed-restricted settings, where other factors become determining of individual growth rate differences, or that the cost of increased social interactions outweighs the stress-reducing effects in HP individuals. It is further possible that the difference between the results of Marin et al. $(1999,2003)$ and our findings is influenced by the main stressors experienced by the birds. Although the stress of living in a large-scale flock may be 1 of the major stressors in the life of an ad libitum-fed broiler, feed restriction can be expected to be the main cause of stress in broiler breeders, and although social support can successfully buffer social stress in the first case, it is probably much less effective at alleviating restriction-induced stress (Rault, 2012).

Another potential explanation for heterogeneity in postnatal growth rates is variability in the development of thermocompetence. A close correlation between initiation of growth in hatchlings and an increased body temperature throughout the first $3 \mathrm{~d}$ of life has been reported previously (Peebles et al., 2004), and the expectation would be that growth, body temperature, and metabolic rate undergo synchronized increases in the postnatal chick, possibly in response to increased circulating levels of triiodothyronine $\left(\mathbf{T}_{\mathbf{3}}\right)$. Chicks that develop thermocompetence earlier would thus have a head start also in terms of growth. Although our results 

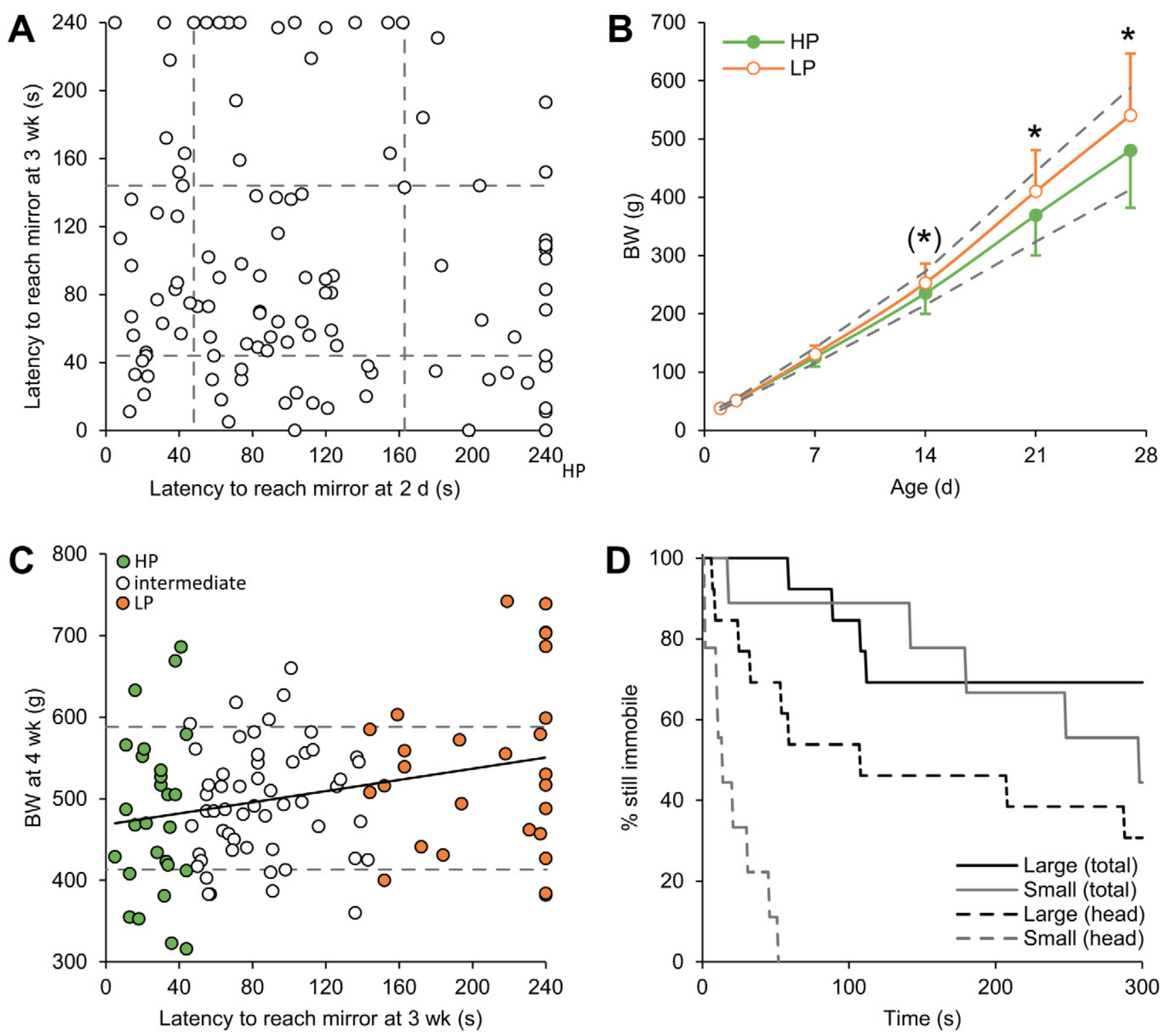

Figure 5. (A) Performance in the sociality T-maze at $2 \mathrm{~d}$ does not predict performance in the same test at $3 \mathrm{wk}$. Dashed lines show the $25 \%$ cutoffs for categorizing animals as high or low performers at $2 \mathrm{~d}$ and $3 \mathrm{wk}$, respectively. (B) The $25 \%$ most social chicks (HP) at $3 \mathrm{wk}$ are significantly lighter at 3 and $4 \mathrm{wk}(P=0.038, P=0.036)$ than their least social conspecifics (LP). Tarsometatarsal lengths were already significantly longer in LP chicks at $2 \mathrm{wk}$ $(P=0.010$, not shown), and there was a trend for difference in BW at this age $(P=0.065)$. Dashed lines mark the \pm 1 SD limits used for classifying birds as either small or large. An asterisk $\left.*^{*}\right)$ denotes significantly different BW between the 2 groups; error bars denote SD. (C) There is a weak, but significant $\left(r^{2}=0.076, P=0.004\right)$, correlation between latency to reach the mirror at $3 \mathrm{wk}$ and BW at $4 \mathrm{wk}$. Animals categorized as HP and LP are shown by solid green and orange circles, respectively, with open circles identifying intermediate birds. Dashed lines mark the \pm 1 SD limits used for classifying birds as either small or large. (D) Survival curves showing the total duration to righting and latency to move the head during tonic immobility. Small birds were significantly quicker to start moving their heads than large birds $(P=0.001)$.

show both the expected increase in body temperature during the first week of age and a clear circadian pattern, we could not find signs of different body temperatures between small and large animals at any age between 2 and $25 \mathrm{~d}$, and the lack of difference in metabolic rate was confirmed by oxygen consumption measurements at $2.5 \mathrm{wk}$. Our expectation that animals growing to be large would show earlier thermocompetence with higher body temperatures and increased growth that may have been caused by higher levels of circulating $T_{3}$ was thus not supported by our results, although the circadian temperature pattern seen is known to be specific to feed restriction in broiler-type chickens as a result of $\mathrm{T}_{3}$ inactivation (Van der Geyten et al., 1999; Savory et al., 2006). Two main differences in experimental setup may explain the difference between the results of Peebles et al. (2004) and this study: first, Peebles and colleagues could perform temperature measurements at the hatchery, whereas our measurements started later, and second, their measurements were performed under highly varying external temperatures that may have increased differences in body temperature, whereas ours were performed under more consistent conditions. Although we did not find any body temperature differences between 


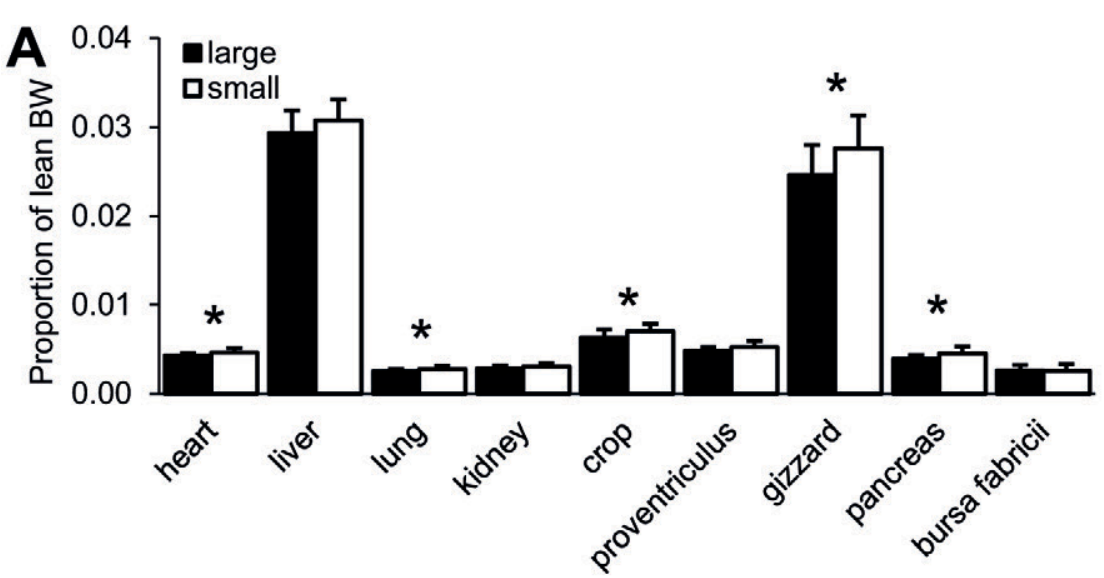

Supply organs at $4 \mathrm{wk}$

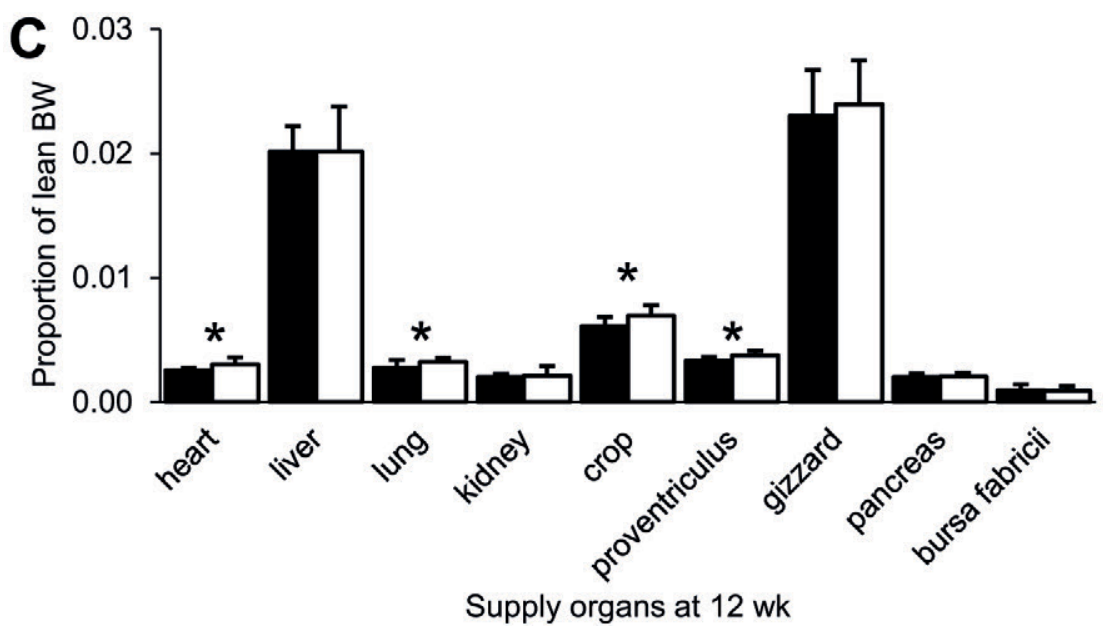

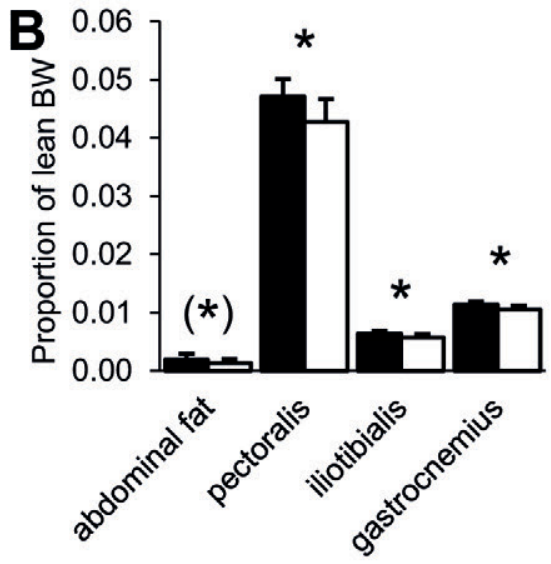

Demand organs at $4 \mathrm{wk}$

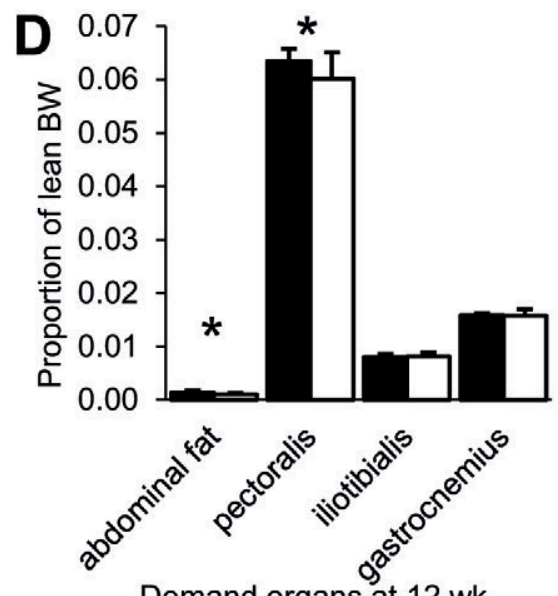

Demand organs at $12 \mathrm{wk}$

Figure 6. (A), (B) Small birds at 4 wk demonstrate relatively larger supply organs (heart, lung, crop, gizzard, and pancreas). In contrast, large birds have larger demand tissues with significantly heavier muscles and a trend for more fat around the gizzard. (large: 13 animals; small: 15 animals). (C), (D) At $12 \mathrm{wk}$, small birds have caught up in relative leg muscle size but still have less fat, smaller pectorals, and significantly larger hearts, lungs, proventriculi, and crops. Although the animals have become more similar in size, the difference between small and large animals in heart and lung size has increased (large: 12 animals, small: 14 animals). Error bars denote SD.

slow- and fast-growing animals, recent studies have shown that different lengths of time between hatching and first access to feed may affect the relationship between body temperature and growth, with low levels of $\mathrm{T}_{3}$ being associated with increased early growth when chicks start feeding soon after hatching but not in initially fasted chicks (Wang et al., 2014, 2016). This suggests a potential confounder that we could not correct for, as all chicks used in this study came from commercial hatcheries that do not record individual hatching times. On the basis of standard commercial procedures and the known transport times between the hatchery and research facility, the animals used to record body temperature are expected to have been fasting for around 12 to $36 \mathrm{~h}$ before first feeding.

We found no predictive value of day-old size measurements (BW or MT length) on later growth performance or body composition within hatchlings from the same maternal flock, but higher growth rates during the first 4 wk were significantly correlated with an increased proportion of muscle mass and relatively smaller gastrointestinal organs at $4 \mathrm{wk}$ of age. This agrees with a previous study in which we also hypothesized that this may incur long-term effects on individual performance (Lindholm et al., 2015). Follow-up dissections of 12-wk-old pullets reported here did, however, reveal reduced differences in body composition after grading and promotion of compensatory growth in smaller animals. This suggests that the current rearing practices for broiler breeders not only improve BW uniformity but also reduce heterogeneity in body composition. Only 2 organs did not show a pattern of approaching homogeneity, with the relative mass of the heart and lungs exhibiting greater difference at $12 \mathrm{wk}$ than at $4 \mathrm{wk}$. We expect that this is associated with the higher metabolic rate required during management-promoted compensatory growth in the initially smaller animals and may have beneficial long-term consequences for these 
Table 2. Analysis of variance comparing 4-wk body composition of chicks hatched either small or large ${ }^{1}$

\begin{tabular}{|c|c|c|}
\hline Organ & $\begin{array}{c}\text { Treatment effect } \\
\text { (hatched small or large) }\end{array}$ & $\begin{array}{l}\text { Covariate effect } \\
\text { (4-wk BW) }\end{array}$ \\
\hline Abdominal fat & ns & $F=4.36, P=0.043$ \\
\hline Pectoralis & ns & $F=10.27, P=0.003$ \\
\hline Iliotibialis & ns & $F=14.27, P<0.001$ \\
\hline Gastrocnemius & ns & $F=19.17, P<0.001$ \\
\hline Ventricular heart & ns & ns \\
\hline Liver & ns & ns \\
\hline Left lung & ns & ns \\
\hline Left kidney & ns & ns \\
\hline Crop & ns & ns \\
\hline Proventriculus & $(F=3.14, P=0.084)$ & ns \\
\hline Gizzard & $(F=3.54, P=0.067)$ & $F=6.49, P=0.015$ \\
\hline Pancreas & ns & $F=9.09, P=0.004$ \\
\hline Bursa fabricii & ns & ns \\
\hline Spleen & ns & ns \\
\hline Duodenum (length) & ns & $F=6.36, P=0.016$ \\
\hline Jejunum (length) & ns & $(F=3.42, P=0.072)$ \\
\hline Ileum (length) & ns & $(F=3.26, P=0.078)$ \\
\hline Avg cecum (length) & ns & $\mathrm{ns}$ \\
\hline Large intestine (length) & ns & ns \\
\hline Total small intestine (length) & ns & $F=4.89, P=0.033$ \\
\hline Total intestine (length) & ns & $F=5.12, P=0.029$ \\
\hline \multicolumn{3}{|c|}{$\begin{array}{l}{ }^{1} \text { The test used absolute organ masses, with lean BW at } 4 \mathrm{wk} \text { as a covari- } \\
\text { ate. The comparison includes all females culled at } 4 \mathrm{wk}, 22 \text { hatched small } \\
\text { ( } 30.4 \text { to } 33.1 \mathrm{~g} \text { ) and } 23 \text { hatched large ( } 38.6 \text { to } 41.3 \mathrm{~g} \text { ). Lean BW at } 4 \mathrm{wk} \\
\text { were } 310.8 \text { to } 571.1 \mathrm{~g} \text { for the small-at-hatch group and } 308.1 \text { to } 647.5 \mathrm{~g} \\
\text { for the large-at-hatch group. More details on BW of these animals can be } \\
\text { found in Fig. 3D. Results } 0.1>P>0.05 \text { are shown in parentheses. ns }=\text { not } \\
\text { significant }(P>0.1) \text {. }\end{array}$} \\
\hline
\end{tabular}

animals if maintained into the laying phase. Although slow-growing broiler breeders may intuitively appear to be poor performers in a production scenario, these results suggest that this is not necessarily the case when uniformity-improving practices are employed.

\section{Conclusions}

Feed restriction is a necessary but problematic practice in the rearing of broiler breeders, both giving rise to animal welfare concerns and complicating animal management because of increased variance in growth. The results of this study suggest that the basis of growth heterogeneity is largely settled before feed amounts become restrictive, which may explain why different rearing practices aimed at reducing growth heterogeneity by reducing feed competition are only partially effective. As heterogeneous growth patterns appear to be present already within a day of starting feeding, we suggest that these may be dependent on differences already determined before arrival at the rearing farm, e.g., because of variability in hatching time (and thus early fasting time) or in maternal investment in the egg.
Understanding when and how these differences arise may help us develop new strategies to improve growth uniformity and broiler breeder management.

\section{APPENDIX 1. DETERMINATION OF THE THERMONEUTRAL ZONE IN 2-WK-OLD BROILER CHICKENS}

Broiler chickens (Ross 308) between 14 and 17 $\mathrm{d}$ of age were used in the determination of the thermoneutral zone (TNZ) by measuring metabolic rate at different environmental temperatures. Metabolic rates were measured as detailed in the main text. Briefly, oxygen consumption was measured overnight in climatic respiration chambers. Four animals were individually placed in 3-L respiration chambers, and measurements were done overnight (1700 to 0700 h) in a temperature-controlled chamber (Rumed Cooled Incubator Model 3001, Rubarth Apparate $\mathrm{GmBH})$. Temperatures were continuously measured using 4-wire Resistance Temperature Detectors of the Pt100 type (model 7410000, Pentronic AB, Gunnebo, Sweden) custom fitted to the lid of each respirometer. Gas from the 4 respiration chambers was analyzed using an oxygen analyzer (FOXBOX II, Sable Systems) for $2.5 \mathrm{~min}$ each, resulting in 1 measurement for each animal every $10 \mathrm{~min}$. Before entering the gas analyzer, the air passed through Drierite (calcium sulfate with cobalt chloride; Hammond Drierite, Xenia, $\mathrm{OH}$ ) to remove water vapor. Baseline oxygen levels were recorded between every chamber. Oxygen consumption was calculated from the standard equation in the absence of $\mathrm{CO}_{2}$ scrubbing (Eq. [10.2] in Lighton, 2008).

The most common experimental procedure to determine the TNZ is to subject the animals to progressively increasing or decreasing temperatures at fixed rates of temperature change, commonly $1^{\circ} \mathrm{C}$ to $2^{\circ} \mathrm{C} / \mathrm{h}$ (Meltzer, 1983; Withers et al., 1987). The same procedure was used here, and the results are shown in Fig. A1C. Runs below an expected lower critical temperature (LCT runs) were initiated at $32^{\circ} \mathrm{C}$ and progressively decreased overnight every $2 \mathrm{~h}$ in steps of $2^{\circ} \mathrm{C} / \mathrm{h}$ to a nominal temperature of $18^{\circ} \mathrm{C}$ (which resulted in a real chamber temperature of $20.5^{\circ} \mathrm{C}$ because of the heat production of the animals being measured). Runs above an expected upper critical temperature (UCT runs) were initiated at $30^{\circ} \mathrm{C}$ and progressively increased overnight every $2 \mathrm{~h}$ in steps of $2^{\circ} \mathrm{C} / \mathrm{h}$ up to $36^{\circ} \mathrm{C}$ and then $1{ }^{\circ} \mathrm{C} / \mathrm{h}$ up to $42^{\circ} \mathrm{C}$. Eight chickens were measured in LCT runs, and 14 chickens were measured in UCT runs.

To account for possible carryover effects, we also devised an experimental protocol in which the climatic chamber was programmed to switch pseudorandomly between different temperatures with an interval of $2 \mathrm{~h}$ 

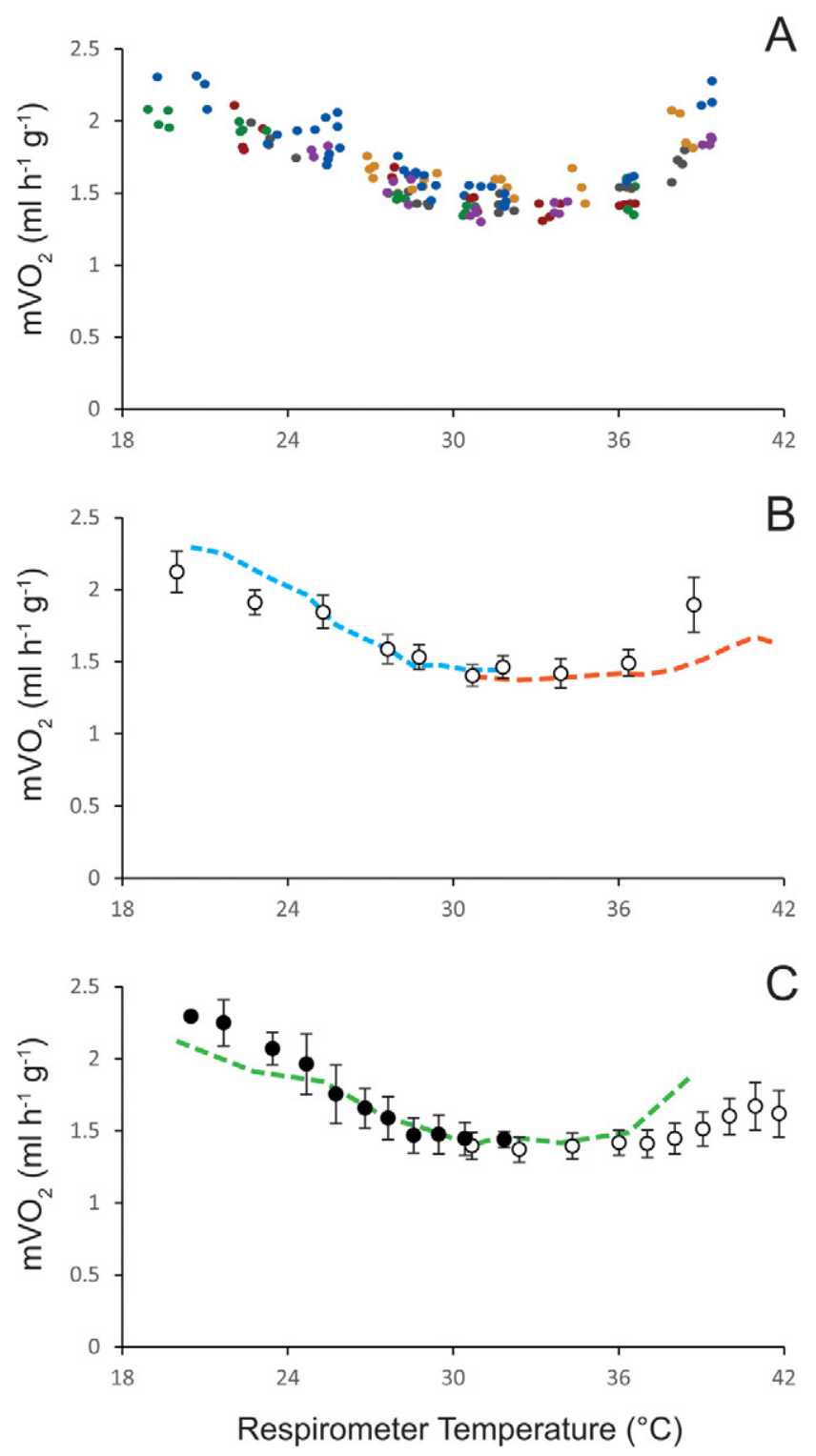

Figure A1. A) Results from the pseudorandom temperature exposure with color codes indicating each of the experimental runs. B) Averaged data from the pseudorandom experiment (with SD). Dashed line shows results from the lower critical temperature (LCT) runs in blue and from the upper critical temperature (UCT) runs in red for comparison. C) Averaged data (with SD) from the LCT and UCT runs with dashed line showing pseudorandom experiment for comparison.

at each temperature. The climatic chamber switched between 7 temperatures during 1 run. The climatic chamber was programmed each day with the same temperature profile (4 intermediate temperatures and 3 high or low temperatures). The first and last temperatures during a run were always an intermediate temperature of either $27^{\circ} \mathrm{C}$ or $30^{\circ} \mathrm{C}$. Because of this setting, every other temperature during the run was an intermediate temperature $\left(27^{\circ} \mathrm{C}\right.$ or $\left.30^{\circ} \mathrm{C}\right)$ with a high $\left(33^{\circ} \mathrm{C}, 36^{\circ} \mathrm{C}\right.$, or $\left.39^{\circ} \mathrm{C}\right)$ or low temperature $\left(18^{\circ} \mathrm{C}, 21^{\circ} \mathrm{C}\right.$, or $\left.24^{\circ} \mathrm{C}\right)$ in between. The experimental protocol was repeated 7 times with 4 animals in each run for a total of 28 individuals.
Basal metabolic rate was obtained from the average oxygen consumption value over the last $30 \mathrm{~min}$ from each programmed temperature. All values are presented as mass-specific oxygen consumption $\left(\mathrm{mL} \mathrm{O}_{2} \cdot \mathrm{g}^{-1} \cdot \mathrm{h}^{-1}\right)$.

As shown in Fig. A1, the TNZ ranged between $30^{\circ} \mathrm{C}$ and $36^{\circ} \mathrm{C}$ regardless of which experimental protocol was used. The results from the pseudorandom temperature exposure are shown in Fig. A1A with color codes indicating each of the experimental runs. Averaged data from this experiment are shown in Figure A1B (mean and SD), and the dashed lines for the LCT (blue) and UCT runs (red) are shown for comparison. The averaged values for LCT and UCT runs are shown in Fig. A1C, with the dashed line showing the pseudorandom data for comparison.

On the basis of these results, a temperature of $32^{\circ} \mathrm{C}$ was shown to determine basal metabolic rate in the main study.

\section{LITERATURE CITED}

Aviagen. 2013. Ross parent stock management handbook: Ross 308. Aviagen, Midlothian, UK.

Cousineau, D. 2011. Randomization test of mean is computationally inaccessible when the number of groups exceeds two. Tutor. Quant. Methods Psychol. 7(1):15-18. doi:10.20982/ tqmp.07.1.p015

Decuypere, E., and V. Bruggeman. 2007. The endocrine interface of environmental and egg factors affecting chick quality. Poult. Sci. 86:1037-1042. doi:10.1093/ps/86.5.1037

Joseph, N. S., A. Lourens, and E. T. Moran Jr. 2006. The effects of suboptimal eggshell temperature during incubation on broiler chick quality, live performance, and further processing yield. Poult. Sci. 85:932-938. doi:10.1093/ps/85.5.932

Kikusui, T., J. T. Winslow, and Y. Mori. 2006. Social buffering: Relief from stress and anxiety. Philos. Trans. R. Soc. B 361:2215-2228. doi:10.1098/rstb.2006.1941

Lew, M. J. 2008. On contemporaneous controls, unlikely outcomes, boxes and replacing the 'Student': Good statistical practice in pharmacology, problem 3. Br. J. Pharmacol. 155:797-803. doi:10.1038/bjp.2008.350

Lighton, J. R. B. 2008. Measuring metabolic rates. A manual for scientists. Oxford Univ. Press, New York. doi:10.1093/acprof:o so/9780195310610.001.0001

Lilburn, M. S., and S. Loeffler. 2015. Early intestinal growth and development in poultry. Poult. Sci. 94:1569-1576. doi:10.3382/ps/ pev104

Lindgren, I., and J. Altimiras. 2013. Prenatal hypoxia programs changes in $\beta$-adrenergic signaling and postnatal cardiac contractile dysfunction. Am. J. Physiol. Regul. Integr. Comp. Physiol. 305:R1093-R1101. doi:10.1152/ajpregu.00320.2013

Lindholm, C., A. Calais, J. Jönsson, N. Yngwe, E. Berndtson, E. Hult, and J.Altimiras. 2015. Slow and steady wins the race? No signs of reduced welfare in smaller broiler breeder hens at four weeks of age. Anim. Welf. 24:447-454. doi:10.7120/09627286.24.4.447

Løtvedt, P., and P. Jensen. 2014. Effects of hatching time on behavior and weight development of chickens. PLoS One 9(7):e103040. doi:10.1371/journal.pone. 0103040 
Marin, R. H., R. B. Jones, D.A. Garcia, andA.Arce. 1999. Early T-maze behavior and subsequent growth in commercial broiler flocks. Br. Poult. Sci. 40(4):434-438. doi:10.1080/00071669987160

Marin, R. H., D. G. Satterlee, S. A. Castille, and R. B. Jones. 2003. Early T-maze behavior and broiler growth. Poult. Sci. 82:742748. doi: $10.1093 / \mathrm{ps} / 82.5 .742$

Meltzer, A. 1983. Thermoneutral zone and resting metabolic rate of broilers. Br. Poult. Sci. 24:471-476. doi:10.1080/00071668308416763

Noy, Y., and D. Sklan. 1999. Energy utilization in newly hatched chicks. Poult. Sci. 78:1750-1756. doi:10.1093/ps/78.12.1750

Peebles, E. D., R. W. Keirs, L. W. Bennett, T. S. Cummings, S. K. Whitmarsh, and P. D. Gerard. 2004. Relationships among posthatch physiological parameters in broiler chicks hatched from young breeder hens and subjected to delayed brooding placement. Int. J. Poult. Sci. 3(9):578-585. doi:10.3923/ijps.2004.578.585

Pinchasov, Y. 1991. Relationship between the weight of hatching eggs and subsequent performance of broiler chicks. Br. Poult. Sci. 32(1):109-115. doi:10.1080/00071669108417332

Rault, J.-P. 2012. Friends with benefits: Social support and its relevance for farm animal welfare. Appl. Anim. Behav. Sci. 136:114. doi:10.1016/j.applanim.2011.10.002

Savory, C. J., L. Kostal, and I. M. Nevison. 2006. Circadian variation in heart rate, blood pressure, body temperature and EEG of immature broiler breeder chickens in restricted-fed and ad libitum-fed states. Br. Poult. Sci. 47(5):599-606. doi:10.1080/00071660600939719

Siegel, P. B., C. F. Honaker, and W. M. Rauw. 2008. Selection for high production in poultry. In: W. M. Rauw, editor, Resource allocation theory applied to farm animal production. CABI Publ., Wallingford,UK.p. 230-242. doi:10.1079/9781845933944.0230

Smith, H. G., and K.-J. Wettermark. 1995. Heritability of nestling growth in cross-fostered European starlings Sturnus vulgaris. Genetics 141(2):657-665.

Tona, K., F. Bamelis, B. De Ketelaere, V. Bruggeman, V. M. B. Moraes, J. Buyse, O. Onagbesan, and E. Decuypere. 2003. Effects of egg storage time on spread of hatch, chick quality, and chick juvenile growth. Poult. Sci. 82:736-741. doi:10.1093/ps/82.5.736
Van der Geyten, S., E. Van Rompaey, J. P. Sanders, T. J. Visser, E. R. Kühn, and V. M. Darras. 1999. Regulation of thyroid hormone metabolism during fasting and refeeding in chicken. Gen. Comp. Endocrinol. 116:272-280. doi:10.1006/gcen.1999.7368

Vieira, S. L., and E. T. Moran Jr. 1998. Broiler chicks hatched from egg weight extremes and diverse breeder strains. J. Appl. Poult. Res. 7(4):392-402. doi:10.1093/japr/7.4.392

Wang, Y., J. Buyse, Z. Song, E. Decuypere, and N. Everaert. 2016. AMPK is involved in the differential neonatal performance of chicks hatching at different time. Gen. Comp. Endocrinol. 228:53-59. doi:10.1016/j.ygcen.2016.02.008

Wang, Y., E. Willems, H. Willemsen, L. Franssens, A. Koppenol, X. Guo, K. Tona, E. Decuypere, J. Buyse, and N. Everaert. 2014. Spread of hatch and delayed feed access affect post hatch performance of female broiler chicks up to day 5. Animal 8(4):610617. doi:10.1017/s175173111400007x

Willemsen, H., N. Everaert, A. Witters, L. De Smit, M. Debonne, F. Verschuere, P. Garain, D. Berckmans, E. Decuypere, and V. Bruggeman. 2008. Critical assessment of chick quality measurements as an indicator of posthatch performance. Poult. Sci. 87:2358-2366. doi:10.3382/ps.2008-00095

Withers, P. C., R. B. Forbes, and M. S. Hedrick. 1987. Metabolic, water and thermal relations of the Chilean Tinamou. Condor 89:424 426. doi: $10.2307 / 1368498$

Wolanski, N. J., R. A. Renema, F. E. Robinson, V. L. Carney, and B. I. Fancher. 2006. Relationships between chick conformation and quality measures with early growth traits in males of eight selected pure or commercial broiler breeder strains. Poult. Sci. 85:1490-1497. doi:10.1093/ps/85.8.1490

Zuidhof, M. J., D. E. Holm, R. A. Renema, M. A. Jalal, and F. E. Robinson. 2015. Effects of broiler breeder management on pullet body weight and carcass uniformity. Poult. Sci. 94:13891397. doi: $10.3382 / \mathrm{ps} /$ pev064

Zuidhof, M. J., F. E. Robinson, J. J. R. Feddes, and R. T. Hardin. 1995. The effects of nutrient dilution on the well-being and performance of female broiler breeders. Poult. Sci. 74(3):441-456. doi:10.3382/ps.0740441 